\title{
Mass cholera vaccination finally possible
}

D ecades after the first successful trials of an oral cholera vaccine, Bangladesh and other developing countries are now considering vaccinating those most at risk of the disease.

Cholera strikes some three to five million people annually and can kill in a matter of hours if left untreated. Spread through contaminated feces, outbreaks are most virulent where proper sanitation is most difficult: slums, conflict zones and areas crippled by natural disaster.

The oral vaccine Dukoral has been internationally available since 1991, but its use has largely been confined to wealthy travellers. In fact, until recently, public health experts feared that widescale vaccination would detract from efforts to improve living conditions in the developing world.

Now that those efforts have failed to curb the disease, attitudes are changing, as has the role of vaccine scientists, says Dr. John Clemens, executive director of icddr,b (International Centre for Diarrhoeal Disease Research, Bangladesh).

"Scientists of my generation feel very conflicted about being advocates," he told CMAJ. But the 30-year saga to prove the merit of cholera vaccination shows that, in some cases, "the evidence doesn't always speak for itself."

In 1983, Clemens led the first largescale field trial of the oral vaccine, later licensed as Dukoral, alongside a simplified form of the vaccine lacking the B-subunit cholera toxin. He reports in a 1986 article in The Lancet that Dukoral was more effective in the short term, affording $85 \%$ protection against cholera after six months, whereas the simplified vaccine provided less protection (58\%). In a 1990 follow-up article, both vaccines showed comparable protection of about $50 \%$ over longer periods.

Although Dukoral was safe and the most effective, it was too expensive for public heath programs in developing countries and it needed to be taken with clean water, which was a "logistic hassle" in cholera-prone areas, says Clemens.

He helped Vietnam develop a more

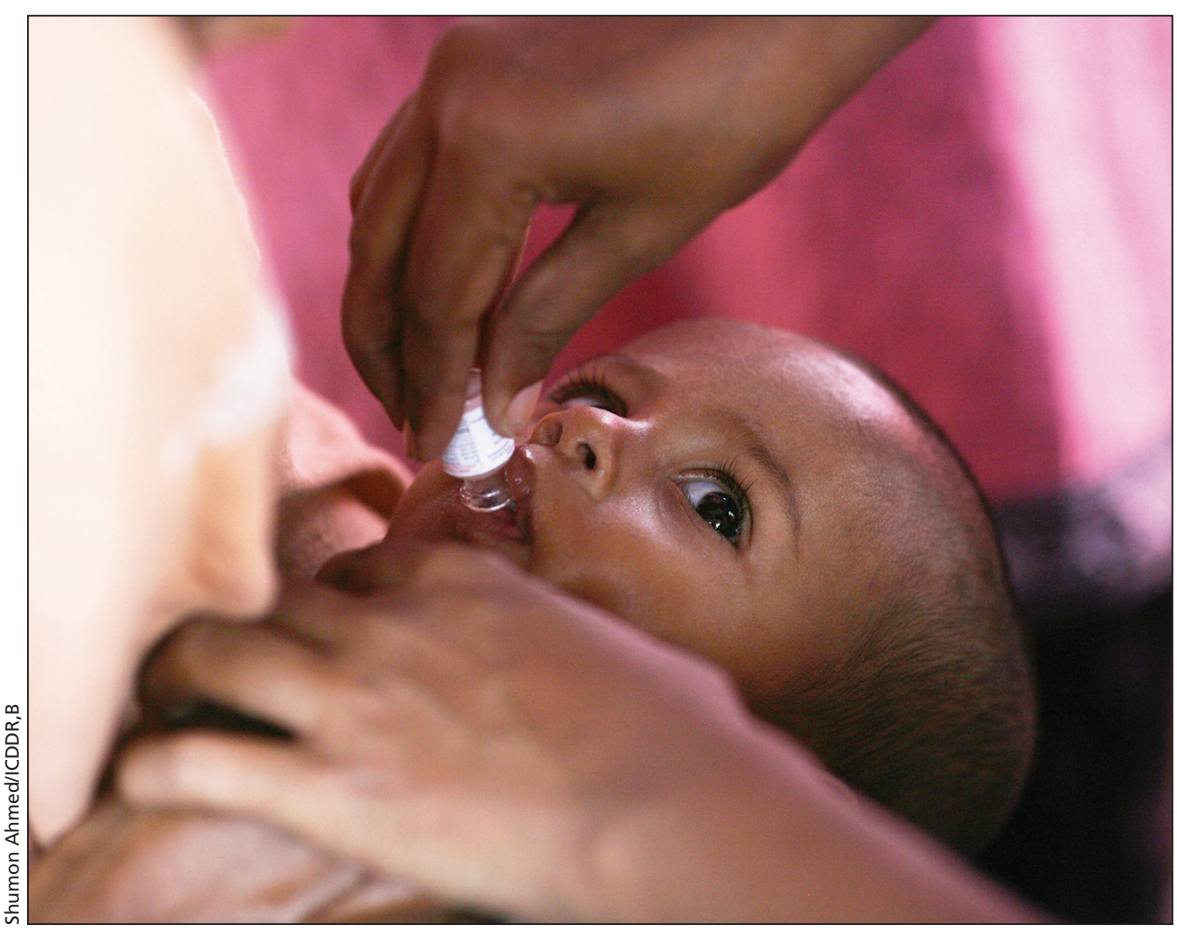

Oral cholera vaccines were proven safe and effective in the 1980 s, so why are they only being used now?

effective version of the simplified vaccine in 1997. That vaccine was cheaper and easier to produce than Dukoral and could be taken without a liquid buffer. But like Dukoral, it never took off internationally because Vietnam's regulatory agency isn't recognized by the World Health Organization (WHO).

"If you're thinking about vaccines for the poor, the most likely buyers are United Nations agencies, and without WHO pre-qualification, they can't purchase the vaccine," explains Clemens.

Then in 1999, Clemens became director-general of the new International Vaccine Institute in South Korea. He used the organization's first grant to reformulate the Vietnamese vaccine to international standards. Following a large and successful field trial in India, a local biotech company, Shantha, licensed the modified vaccine under the name Shanchol in 2009.

The vaccine was then eligible for WHO pre-qualification, but licensure in India turned out to be a case of "winning the battle but not the war," says Clemens. Oral cholera vaccine wasn't a high priority for WHO or most public health programs. Myriad objections to cholera vaccination prevailed; key among them was that prevention efforts should focus on improving access to clean water and sanitation.

"It was like arguing that you shouldn't give an AIDS vaccine because it will embolden people to have sex without precaution," says Clemens. "It was really a straw-man argument because we had lots of experience and we never found that giving the vaccine had a punitive effect [on other prevention efforts]."

Two things eventually turned the tide of opinion in favour of vaccination. The first was a growing body of evidence of the additional benefits of vaccination. Notably, a reanalysis of trial data from the 1980s published in The Lancet in 2005 , showed that vaccination could provide indirect protection or "herd immunity" for the unvaccinated. Later studies also showed that it was less 
expensive to vaccinate than to treat cholera, and that it was feasible for public health programs to deliver the vaccine using existing infrastructure.

"Years ago the vaccine community was mostly focused on clinically evaluating vaccines ... but what I learned from this story was you need this very practical evidence, and even that may not be enough," says Clemens.

In some cases, unfortunately, it also takes a disaster to spur action, he notes. "Undoubtedly one of the main reasons for growing acceptance of cholera vaccination was the horrible epidemic in
Haiti following the 2010 earthquake." In 2011, the World Health Assembly urged the use of oral vaccines in outbreaks and areas with endemic cholera, and Shanchol received WHO pre-qualification. WHO has since stockpiled 2 million doses of the vaccine, and the $\mathrm{UN}$ has incorporated vaccination in its 2014-15 strategy for curbing cholera in Haiti and the Dominican Republic.

icddr,b is hopeful that Bangladesh will roll out a mass vaccination campaign in the next three years, after the collection of additional data necessary for a countrywide commitment. Last year, the organization partnered with Dhaka-based Incepta Pharmaceuticals to develop a new, even cheaper vaccine. Meanwhile, experiences in Guinea and Haiti have proven the usefulness of vaccination in outbreak settings.

The next step will be ensuring a global supply of vaccine to meet the 200 million doses required by 2025 , says Clemens. "In Bangladesh, maybe half the population is high risk; that's half of 160 million people, so you can do the math." - Lauren Vogel, CMAJ

CMAJ 2015. DOI:10.1503/cmaj.109-5021 